\title{
Graphene ribbons with a line of impurities: Opening of a gap
}

\author{
R. N. Costa Filho, ${ }^{1,2, *}$ G. A. Farias, ${ }^{1, \dagger}$ and F. M. Peeters ${ }^{3, \sharp}$ \\ ${ }^{1}$ Departamento de Física, Universidade Federal do Ceará, Caixa Postal 6030, Campus do Pici, 60455-760 Fortaleza, Ceará, Brazil \\ ${ }^{2}$ Consortium of the Americas for Interdisciplinary Science, University of New Mexico, Albuquerque, New Mexico 87131, USA \\ ${ }^{3}$ Department of Physics, University of Antwerp, Groenenborgerlaan 171, B-2020 Antwerpen, Belgium \\ (Received 22 May 2007; revised manuscript received 6 August 2007; published 27 November 2007)
}

\begin{abstract}
A tight-binding model is used to study the role of impurities in a graphene ribbon. The graphene ribbon is made of lines of carbon atoms in an armchair or zigzag shape where the impurities are included in the ribbon substitutionally. The addition of a line of impurities opens up a gap in the energy spectrum. The size of it depends in a nonmonotonic way on the position of the impurity line, and also on the strength of the carbonimpurity interaction.
\end{abstract}

DOI: 10.1103/PhysRevB.76.193409

PACS number(s): 81.05.Uw, 73.20.At, 73.20.Mf, 73.21.-b

Graphenes have attracted attention lately due to their potential application in nanotechnological devices and their unusual Dirac energy spectrum. Although the theoretical aspects were studied more than 50 years ago, ${ }^{1,2}$ it was only three years ago, after the first experimental results, that this system became a hot topic in material science and condensed matter physics. ${ }^{3-7}$

Graphene is a zero-gap material which, together with its linear energy spectrum, leads to the absence of confinement by electric potentials. ${ }^{8}$ In order for gating to be used as an effective way to manipulate the electron mobility, one needs a way to open an energy gap around the $K$ and $K^{\prime}$ points.

Recently, it was shown that in bilayer graphene it is possible to create a gap at the $K$ point by using chemical doping or by the application of an electric field perpendicular to the bilayer, i.e., by applying a gate potential. ${ }^{9-12}$ A similar approach does not work for a single graphene layer and it is not clear how one can create an energy gap at the $K$ point. The electronic properties of these graphene layers or ribbons are strongly dependent on their geometry or size. ${ }^{13-20}$ Some attempts to tune the electronic properties of these ribbons are focusing on the study of chemical edge modifications, ${ }^{19}$ application of uniaxial strain, ${ }^{20}$ and boron-doped graphene ribbons, ${ }^{21}$ where a substitutional boron atom is introduced at some specific sites of the graphene lattice.

In the present paper, we show that the introduction of a line of impurities can also do the job of tuning the electronic properties of a graphene ribbon. This approach to graphene ribbons opens the possibility for a variety of energy spectra according to the position and strength of the impurity line. The system is defined as a honeycomb lattice of carbon atoms with two sublattices $A$ and $B$. The graphene sheet is infinite in the $x$ direction and has a limited number of carbon lines in the $y$ direction with $N$ atom lines. The impurities are introduced substitutionally where we choose a carbon line and exchange it for a different one, silicon or boron for example (see Fig. 1). At present, it is not clear how to produce such a line of impurities, but one hopes that future advances in graphene growth will allow for such atomic scale positioning of impurities. Our model is based on a microscopic approach using a tight-binding Hamiltonian as follows:

$$
H=-\sum_{i, j} t_{i j} a_{i}^{\dagger} b_{j}+\text { H.c. }
$$

where we consider, for simplicity, that the hopping term does not change near the ribbon surface, and is defined as $t_{i j}=t$ for electrons in sublattice $A$ hopping to its neighbors in sublattice $B$, and vice versa. When the electron hops to the impurity sites we define $t_{i j}=t_{I}$, and we consider that the impurityimpurity hopping $t_{I I}=t_{I}$. The operators $a_{i}^{\dagger}\left(a_{i}\right)$ create (annihilate) an electron on site $i$ in sublattice $A$ and the operators $b_{i}^{\dagger}\left(b_{i}\right)$ act on sublattice $B$. The operators $a^{\dagger}(a)$ and $b^{\dagger}(b)$ depend on the one-dimensional (1D) wave vector $q=q_{x}$ parallel to the ribbon surface and the indices $n$ and $n^{\prime}$ $(=1,2, \ldots, N)$ that label the carbon and impurity lines parallel to the surface. We can rewrite the Hamiltonian as

$$
H=-\sum_{q_{x} n n^{\prime}}\left[\tau_{n n^{\prime}}\left(q_{x}\right) a_{q_{x} n^{\dagger}}^{\dagger} b_{q_{x} n^{\prime}}+\text { H.c. }\right] .
$$

For a Hamiltonian which contains only quadratic operators of fermions or bosons, the operator statistics are irrelevant. Therefore, the same eigenvalues are obtained for fermions or bosons, and the operators can be considered creation and annihilation operators of a harmonic oscillator. Then, in order to find the band structure of the system along the Brillouin zone we have to diagonalize the Hamiltonian to find the eigenvalues of the system (see, for example, Ref. 22). We apply the standard equation of motion $i \hbar d A / d t$ $=[A, H]$ (for any operator $A$ ) to the operators $a_{q_{x} n}$ and $b_{q_{x} n}$ in line $n$. Taking $\hbar=1$ and assuming that the modes behave like

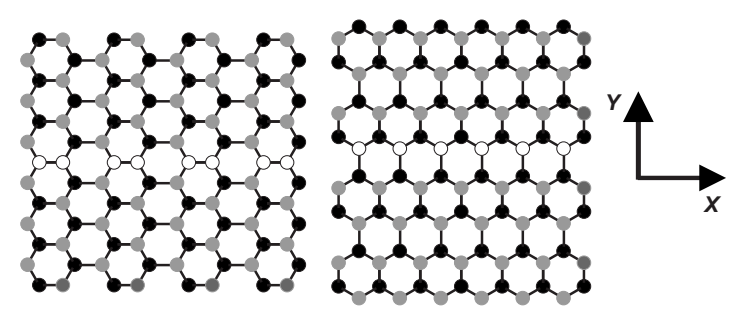

FIG. 1. A graphene ribbon where black (gray) dots are the sublattice $A(B)$ with a line of impurities (white dots) in the middle of the sheet. 
TABLE I. Value of the matrix elements in the hopping matrix.

\begin{tabular}{lcl}
\hline \hline Parameter & Zigzag & Armchair \\
\hline$\alpha$ & 0 & $t e^{-i q_{x} a}$ \\
$\beta$ & $2 t \cos \left(\sqrt{3} q_{x} a / 2\right)$ & $t e^{i q_{x} a / 2}$ \\
$\gamma$ & $t$ & $t e^{i q_{x} a / 2}$ \\
\hline \hline
\end{tabular}

$\exp \left[-i \omega\left(q_{x}\right) t\right]$ we will have the sets of coupled equations

$$
\begin{aligned}
& \omega\left(q_{x}\right) a_{q_{x} n}=\sum_{q_{x} n^{\prime}} \tau_{n n^{\prime}}\left(q_{x}\right) b_{q_{x} n^{\prime}}, \\
& \omega\left(q_{x}\right) b_{q_{x} n}=\sum_{q_{x} n^{\prime}} \tau_{n n^{\prime}}^{*}\left(q_{x}\right) a_{q_{x} n^{\prime}} .
\end{aligned}
$$

There are $2 N$ equations where the amplitude factors $\tau_{n n^{\prime}}\left(q_{x}\right)$ are given by

$$
\tau_{n n^{\prime}}\left(q_{x}\right)=t\left[\exp \left(i q_{x} a\right) \delta_{n^{\prime}, n}+\exp \left(i \frac{1}{2} q_{x} a\right) \delta_{n^{\prime}, n \pm 1}\right]
$$

for the armchair configuration, and

$$
\tau_{n n^{\prime}}\left(q_{x}\right)=t\left[2 \cos \left(\frac{\sqrt{3}}{2} q_{x} a\right) \delta_{n^{\prime}, n \pm 1}+\delta_{n^{\prime}, n \mp 1}\right]
$$

for the zigzag case, where the \pm sign depends on the sublattice since the atom line alternates from $A$ and $B$. The above equations can be written in matrix form as

$$
\omega\left(q_{x}\right)\left[\begin{array}{c}
a_{q_{x} n} \\
b_{q_{x} n}
\end{array}\right]=\left[\begin{array}{cc}
0 & T\left(q_{x}\right) \\
T^{*}\left(q_{x}\right) & 0
\end{array}\right]\left[\begin{array}{c}
a_{q_{x} n} \\
b_{q_{x} n}
\end{array}\right],
$$

where the solution of this matrix equation is given by the condition

$$
\operatorname{det}\left[\begin{array}{cc}
-\omega\left(q_{x}\right) I_{N} & T\left(q_{x}\right) \\
T^{*}\left(q_{x}\right) & -\omega\left(q_{x}\right) I_{N}
\end{array}\right]=0,
$$

where $T\left(q_{x}\right)$ is the hopping matrix, which depends on the orientation of the ribbon, and $\omega\left(q_{x}\right)$ are the energies of the modes. The matrix $T\left(q_{x}\right)$ is given by

$$
T=\left[\begin{array}{cccccc}
\alpha & \beta & 0 & 0 & 0 & \ldots \\
\beta & \alpha & \gamma & 0 & 0 & \ldots \\
0 & \gamma & \alpha & \beta & 0 & \ldots \\
0 & 0 & \beta & \alpha & \gamma & \ldots \\
\vdots & \vdots & \vdots & \vdots & \vdots & \ddots
\end{array}\right] .
$$

The parameters $\alpha, \beta$, and $\gamma$ depend on the ribbon geometry and are given in Table I.

Results for a zigzag ribbon are shown in Fig. 2. It shows the dispersion relation for ribbons with 20 and 21 lines of atoms with and without impurities. Depending on the number of lines, the ribbon can present two kinds of energy bands where the main difference is related to the localized modes, since the edges of the zigzag configuration depend on the number of lines. For the zigzag configuration all modes

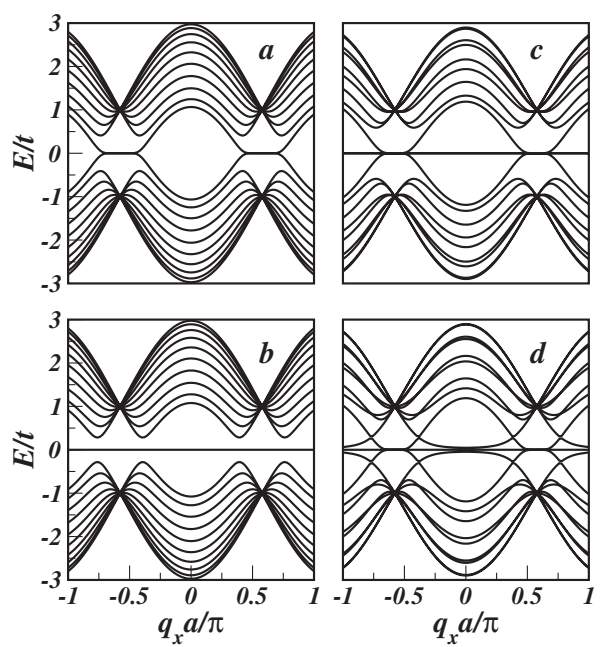

FIG. 2. Dispersion relation for two arrangements of a zigzag ribbon. (a) Energy band for a 20-line ribbon without impurities. (b) Same as in (a) but now the ribbon has 21 lines. (c) A 20-atom-line ribbon with an impurity line at line number 11. (d) Same as in (c) for 21 lines. In (c) and (d) we have used $t_{I}=0$.

are degenerate because of the two sublattices, i.e., we have two modes with the same energy for each sublattice. From Fig. 2(a) one can see degenerate localized modes in the vicinity of $q_{x}= \pm 0.5 \pi / a$, whereas Fig. 2(b) shows these degenerate localized modes for all $q_{x}$ in the Brillouin zone $(E=0)$.

When we add an impurity line in line number 11 for the sample with 20 lines, the band is now modified. The spectrum is still gapless, and is quite different from the one without impurities. The energy band now shows a mixing of two ribbons, one with an odd number of lines and another with an even number of lines. For $t_{I}=0$ we can imagine our system as two ribbons: one with ten lines and another with nine lines. Figure 2(c) can be seen as a superposition of Figs. 2(a) and 2(b) plus the modes related to the impurities. The same can be said about Fig. 2(d), for a ribbon with 21 lines and impurities in line 11. The modes appear as superposition of two ribbons with ten lines. However, they are shifted since the first line of one ribbon belongs to sublattice $A$ while the other ribbon has an atom of sublattice $B$ in the first line of atoms.

Next we analyze the effect of an impurity line on an armchair conformation ribbon. For this geometry we have three different band energy structures depending on the number of lines in the sheet: $3 i, 3 i+1$, and $3 i+2$, where $i=1,2,3, \ldots$. Each of these configurations results in a different energy band. Figure 3 shows the dispersion relation for ribbons with 20, 21, and 22 lines of atoms with and without impurities. Figures 3(a)-3(c) show the usual behavior of dispersion relation for a large armchair ribbon, i.e., the top of the valence band and the bottom of the conduction band are located at $q_{x}=0$. One can see that the energy spectrum of the armchair configuration shows more modes than the one for the zigzag configuration for the same number of lines. The reason is that the two sublattices in the armchair configuration lie on the same line of the ribbon. Therefore the spectrum is not degenerate as in the zigzag case. 

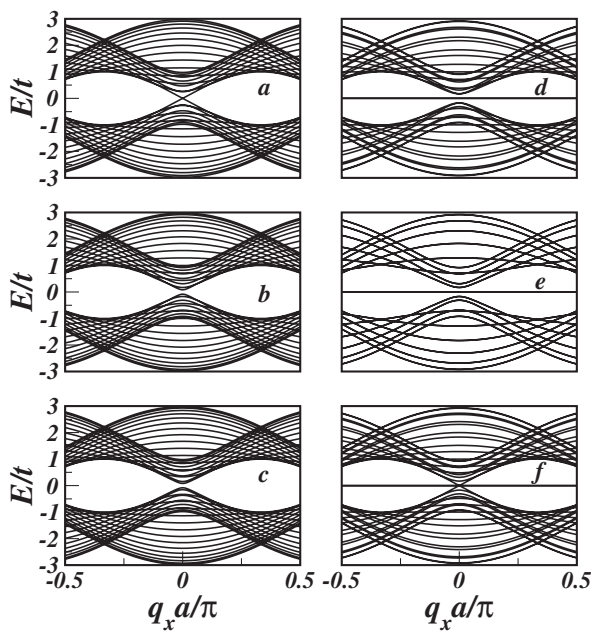

FIG. 3. Energy band for the armchair configuration. (a) A ribbon with 20 lines without impurities. (b) As in (a) but now with 21 atom lines. (c) Same as before for 22 atom lines. (d), (e), and (f) are the energy bands for 20-, 21-, and 22-atom lines, respectively, but now with an impurity in line 11 . Here we took $t_{I}=0$.

When an impurity line is introduced, the energy bands exhibit a degenerate localized mode extending over the whole Brillouin zone (again we are using $t_{I}=0$ ). As in the zigzag case, the energy band is a superposition of two ribbons [see Figs. 3(d)-3(f)]. It is interesting to note that for $N=21$ the energy band is completely degenerate since it is formed by two ribbons with ten lines.

The presence of the impurity line breaks the translational symmetry normal to the edges. Therefore, it creates interface modes decaying away from this line of atoms. This is the main reason for the changes in the spectrum for both the zigzag and armchair configuration. The whole ribbon now behaves like two smaller ribbons coupled through the impurity line. In Fig. 4, we plot the behavior of the energy modes against the ratio between the impurity hopping integral and the hopping term between carbon-carbon atoms $\left(t_{I} / t\right)$ at $q_{x}=0$, for the armchair configuration.

There is one mode starting at the bottom of the conduction band from zero energy, for $t_{I}=0$. The energy of this localized interface mode tends to increase with $t_{I}$. Eventually it interacts and hybridizes with other band modes. This is the same principle for the three armchair configurations studied here. As $t_{I} / t$ approaches 1 the interface mode continues "crossing" the band and the other modes try to accommodate to form the energy band of a single ribbon. Figure 4(a) clearly shows the behavior of the impurity modes. For large values of $t_{I} / t$ the impurity modes leave the "bulk" energy band and increase almost linearly with the impurity hopping integral. Although the figure presents the result for $N=20$, the same behavior is observed for $N=21$ and 22 . The difference between these three ribbons is very distinct when we look for small values of $t_{I} / t$. Figure 4(b) shows, for a ribbon with 20 lines, the impurity mode rising, and also shows the energy modes going to zero as the sample becomes homogeneous. That is expected since for $N=20$ there is no gap in the band for $q_{x}=0$. It is also interesting to note a crossing between electron and hole levels for $t_{I}=t$. For $N=21$ we see
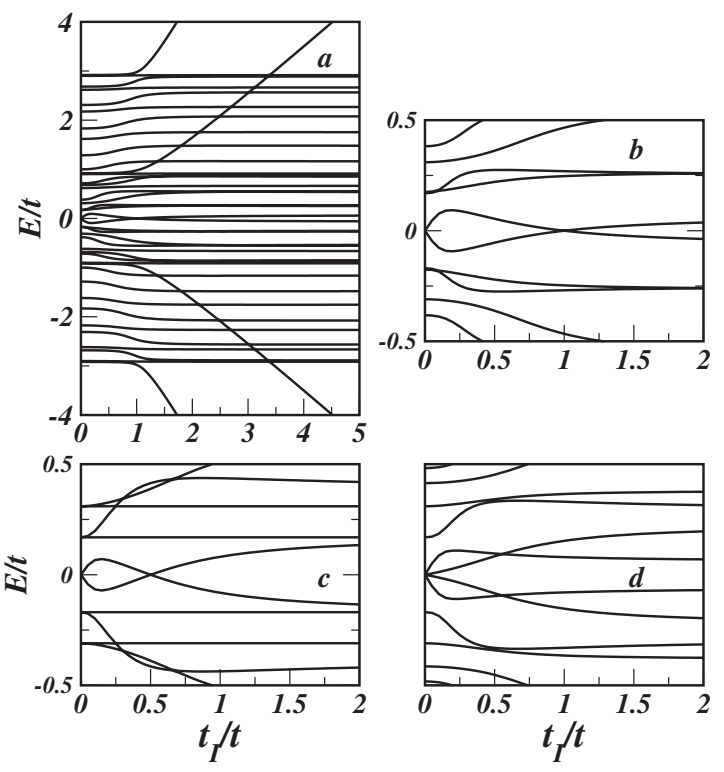

FIG. 4. $q_{x}=0$ dispersion of the modes for a 20-line ribbon with an impurity in the 11th line as a function of the strength of the hopping integral. In the upper left panel all modes of the band are shown, while in the upper right panel we amplified the region around $E=0$. For (c) and (d) we show the lower energy region for $N=21$ and 22 , respectively.

that when $t_{I}=0.5 t$ there is also a crossing between the electron and hole levels [see Fig. 4(c)]. The impurity modes increase faster than in the previous case of $N=20$. When we consider $N=22$, we mentioned above that for $t_{I}=0$ the system behaves like a superposition of two independent ribbons, one with $N=10$ and another with $N=11$ lines. As we know, for $N=3 \times 3+2=11$ the spectrum should have no gap at $q_{x}=0$. Figure $4(\mathrm{~d})$ shows the rising of two modes (which is the impurity one and a normal mode opening the gap).

Figure 5 shows how the gap energy is affected by the impurities. For ribbons with $N=20$ and 21 they behave in a

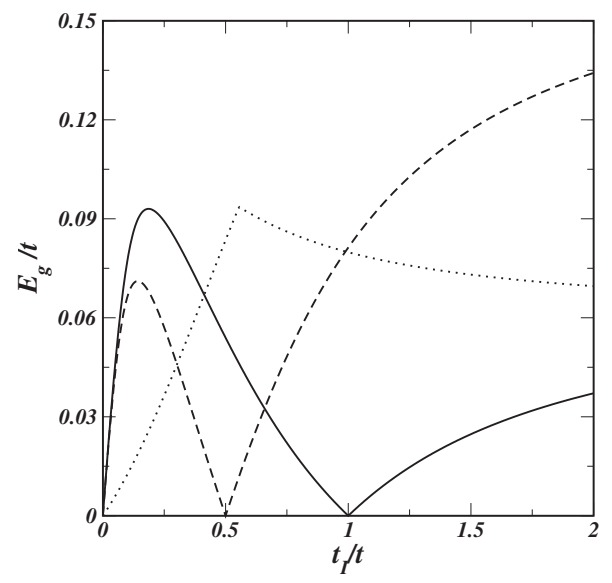

FIG. 5. Variation of the energy gap against the strength of the impurity hopping integral for an armchair ribbon. Solid line for a 20-atom-line ribbon, dashed line for 21 lines, and dotted line for a ribbon with 22 lines of atoms. The impurities are always in line number 11 . 


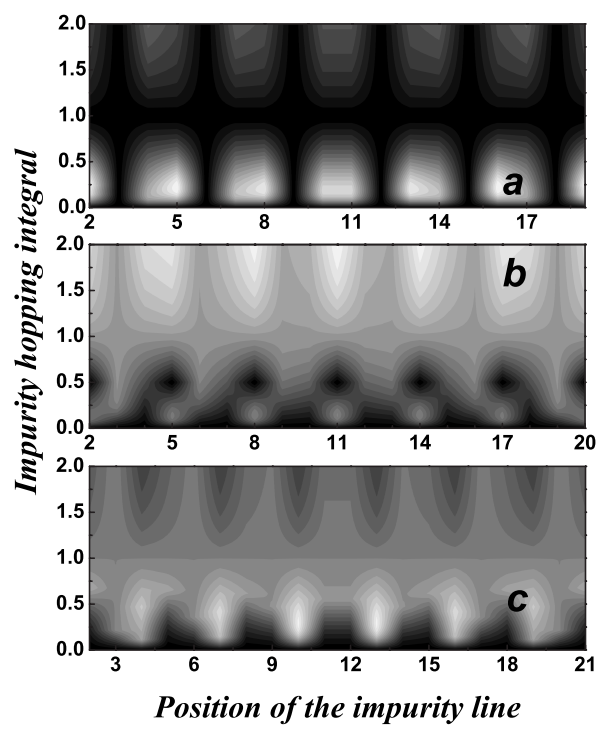

FIG. 6. Contour plot of the energy gap for the armchair ribbon showing the energy gap with the position of the impurity line varying from line 2 to $N-1$ : (a) 20, (b) 21, and (c) 22 lines. The energy increases from black to white.

similar way. They start from zero (the impurity mode at $t_{I}$ $=0$ ) and reach a maximum value (the point of the first crossing with the lowest band mode). Then the gap decreases to zero and increases again. That does not happen for $N=22$. The gap increases until $t_{I} / t=0.5$ and decreases slowly and seems to converge to the same value. The curve is quite different from the two others; this is because, as we could see in Fig. 4, for $N=22$ there is no crossing between electron and hole levels.

In order to make a more general study, we present in Fig. 6 a contour plot of the behavior of the gap energy. We now change the position of the impurity line as well as its "strength." The main feature of this figure is to show that the position of the impurity line in the ribbon is also important and presents the same periodic behavior, i.e., for each three additional lines the spectrum repeats. Here, the introduction of the impurity enriches this behavior, adding the possibility of combinations.

In summary, we have shown here that a simple tightbinding model is able to extract very interesting features of a carbon ribbon when a line of impurities is added to it. The main feature is that controlling the hopping integral of the impurities, which we assumed to be localized on a line through the ribbon, can modify the gap in the energy spectrum. When $t_{I}=0$ the system introduced here can also be understood as a coupling of two graphene ribbons of different sizes separated by some distance $d$. Our results also indicate that the addition of a perturbation in the tight-binding Hamiltonian can open up a gap for the nanoribbon system discussed here.

This work was supported by CNPq (R.N.C.F and G.A.F), the Flemish Science Foundation (FWO-Vl), and the Belgian Science Policy (IAP). *rai@fisica.ufc.br

$\dagger$ gil@fisica.ufc.br

†rancois.peeters@ua.ac.be

${ }^{1}$ P. R. Wallace, Phys. Rev. 71, 622 (1947).

${ }^{2}$ J. C. Slonczewski and P. R. Weiss, Phys. Rev. 109, 272 (1958).

${ }^{3}$ A. K. Geim and K. S. Novosolev, Nat. Mater. 6, 183 (2007).

${ }^{4}$ K. S. Novosolev, A. K. Geim, S. V. Morozov, D. Jiang, Y. Zhang, S. V. Dubonos, I. V. Grigorieva, and A. A. Firsov, Science 306, 666 (2004).

${ }^{5}$ K. S. Novosolev, A. K. Geim, S. V. Morozov, D. Jiang, M. I. Katsnelson, I. V. Grigorieva, S. V. Dubonos, and A. A. Firsov, Nature (London) 438, 197 (2005).

${ }^{6}$ Y. Zhang, Y. Tan, H. L. Stormer, and P. Kim, Nature (London) 438, 201 (2005).

${ }^{7}$ A. H. Castro Neto, Nat. Mater. 6, 176 (2007).

${ }^{8}$ J. M. Pereira, Jr., P. Vasilopoulos, and F. M. Peeters, Appl. Phys. Lett. 90, 132122 (2007).

${ }^{9}$ T. Ohta, A. Bostwick, T. Seyller, K. Horn, and E. Rotenberg, Science 313, 951 (2006).

${ }^{10}$ E. McCann, Phys. Rev. B 74, 161403(R) (2006).
${ }^{11}$ H. Min, B. R. Sahu, S. K. Banerjee, and A. H. MacDonald, Phys. Rev. B 75, 155115 (2007).

${ }^{12}$ J. M. Pereira, Jr., P. Vasilopoulos, and F. M. Peeters, Nano Lett. 7, 946 (2007).

${ }^{13}$ K. Nakada, M. Fujita, G. Dresselhaus, and M. S. Dresselhaus, Phys. Rev. B 54, 17954 (1996).

${ }^{14}$ M. Ezawa, Phys. Status Solidi C 4, 489 (2007).

${ }^{15}$ M. Ezawa, Phys. Rev. B 73, 045432 (2006).

${ }^{16}$ L. Brey and H. A. Fertig, Phys. Rev. B 73, 235411 (2006).

${ }^{17}$ L. Brey and H. A. Fertig, Phys. Rev. B 75, 125434 (2007).

${ }^{18}$ Y.-W. Son, M. L. Cohen, and S. G. Louie, Phys. Rev. Lett. 97, 216803 (2006).

${ }^{19}$ Z. F. Wang, Q. Li, H. Zheng, H. Ren, H. Su, Q. W. Shi, and J. Chen, Phys. Rev. B 75, 113406 (2007).

${ }^{20}$ C. P. Chang, B. R. Wu, R. B. Chen, and M. F. Lin, J. Appl. Phys. 101, 063506 (2007).

${ }^{21}$ T. B. Martins, R. H. Miwa, A. J. R. da Silva, and A. Fazzio, Phys. Rev. Lett. 98, 196803 (2007).

${ }^{22}$ R. N. Costa Filho, M. G. Cottam, and G. A. Farias, Phys. Rev. B 62, 6545 (2000). 